\title{
8
}
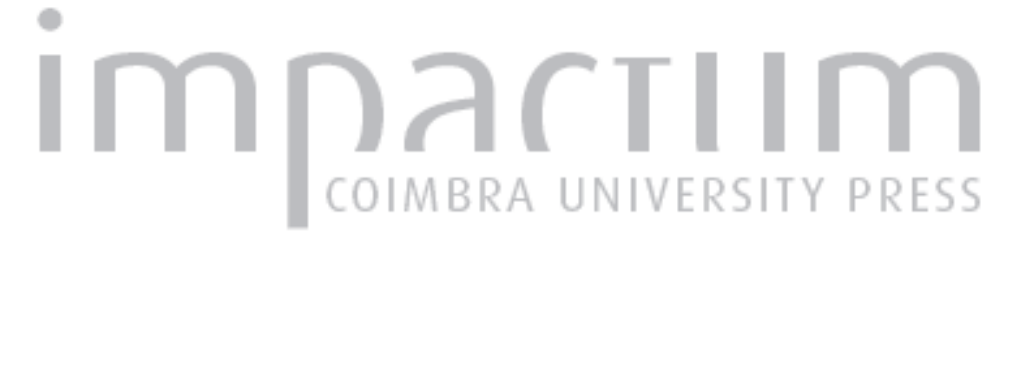

\section{La fabula de Descartes: la libre création des vérités éternell es et le théâtre des Meditationes}

\author{
Autor(es): $\quad$ Gatto, Alfredo
}

Publicado por: Faculdade de Letras da Universidade de Coimbra, Instituto de Estudos

URL

persistente: Filosóficos

DOI: DOI:http://dx.doi.org/10.14195/0872-0851_42_1

Accessed : $\quad$ 26-Apr-2023 09:10:10

A navegação consulta e descarregamento dos títulos inseridos nas Bibliotecas Digitais UC Digitalis, UC Pombalina e UC Impactum, pressupõem a aceitação plena e sem reservas dos Termos e Condições de Uso destas Bibliotecas Digitais, disponíveis em https://digitalis.uc.pt/pt-pt/termos.

Conforme exposto nos referidos Termos e Condições de Uso, o descarregamento de títulos de acesso restrito requer uma licença válida de autorização devendo o utilizador aceder ao(s) documento(s) a partir de um endereço de IP da instituição detentora da supramencionada licença.

Ao utilizador é apenas permitido o descarregamento para uso pessoal, pelo que o emprego do(s) título(s) descarregado(s) para outro fim, designadamente comercial, carece de autorização do respetivo autor ou editor da obra.

Na medida em que todas as obras da UC Digitalis se encontram protegidas pelo Código do Direito de Autor e Direitos Conexos e demais legislação aplicável, toda a cópia, parcial ou total, deste documento, nos casos em que é legalmente admitida, deverá conter ou fazer-se acompanhar por este aviso. 


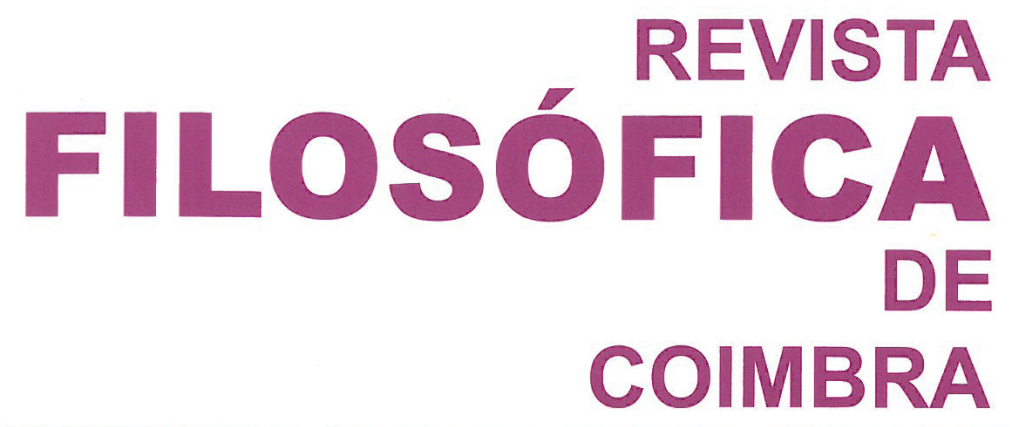

vol. 21 - número 42 - outubro 2012

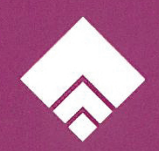




\section{LA FABULA DE DESCARTES. LA LIBRE CRÉATION DES VÉRITÉS ÉTERNELLES ET LE THÉÂTRE DES MEDITATIONES}

\section{ALFREDO GATTO*}

Resume: Dans cet article nous avons essayé de mettre en évidence, à travers une métaphore théâtrale, le rôle que la théorie de la libre création des vérités éternelles a occupé dans la réflexion cartésienne, et nous avons cherché de justifier les raisons pour lesquelles cette théorie n'a pas trouvé place dans les ouvrages publiées par Descartes. À notre avis, il faut chercher la motivation principale précisément dans les présupposés et dans les conséquences de cette doctrine; en effet, la fragilité épistémique résultante de cette théorie n'aurait jamais permis à Descartes de réaliser une description objective et nécessaire du monde. Donc, il est nécessaire d'examiner encore une fois la spéculation cartésienne, en essayant de mettre en lumière les différents lieux textuels où la réflexion de Descartes semble être divisée entre une métaphysique 'publique' et une métaphysique 'privée'.

Mots-clefs: Descartes, Vérités éternelles, Fable, Théâtre, Dieu Trompeur, Malin Génie

Resumo: Neste artigo procurámos salientar, através da metáfora teatral, a importância da teoria sobre a livre criação das verdades eternas na reflexão cartesiana, tentando ao mesmo tempo justificar as razões pelas quais uma tal teoria não encontrou espaço nas obras publicadas por Descartes. Na nossa opinião, a principal motivação pode ser encontrada nos pressupostos e nas consequências dessa mesma doutrina: a fragilidade epistémica resultante desta teoria nunca teria permitido ao filósofo francês dar vida a uma descrição objectiva e necessária do

* Universidade San Rafaelo, Milano. Je voudrais remercier de façon particulaire Mme Giulia Belgioioso et M. Massimiliano Savini pour leurs précieuses indications qui m'ont permis d'améliorer et éclaircir la première rédaction de l'essai. Je voudrais également remercier M. Guy Renard et M. Giacomo Petrarca pour leur aimable assistance dans la rédaction du texte français. 
mundo. É então necessário investigar novamente a especulação cartesiana, tentando elucidar os vários passos onde a reflexão do filósofo parece poder dividir-se entre uma metafísica 'pública' e uma metafísica 'privada'.

Palavras-chave: Descartes, Verdades eternas, Fábula, Teatro, Deus enganador, Génio maligno.

Dans un bref écrit retrouvé parmi les papiers de Leibniz ${ }^{1}$, un Descartes encore jeune semble dégager le noyau le plus profond de sa pensée, le fil rouge qui accompagnera, presqu'en filigrane, l'essor manifeste de son genium spéculatif:

Ut comoedi, moniti ne in fronte appareat pudor, personam induunt: sic ego, hoc mundi theatrum conscensurus, in quo hactenus spectator exstiti, larvatus prodeo (AT, X, p. 213)2.

La comparaison faite sienne par le philosophe français est de nature théâtrale, et les termes utilisés, pris dans leur sens le plus fort, nous offrent un scenario assez détaillé. Descartes, en réalité, tandis qu'il construit sa corrélation imagée et l'énonce au pluriel, ne dit pas actor, mais plutôt comoedus, situant les masques de sa propre future dramaturgie dans le cadre d'un genre bien précis et déterminé. Le passage cité suggère en outre que les personnages qui franchiront le seuil de ce théâtre mondain [mundi theatrum] laisseront dans l'ombre l'auteur de l'entière comédie. Cette simple trace, imprimée et laissée par celui qui en a eu l'idée, se présentera à nouveau sur scène, après une longue et tourmentée gestation, dans l'incipit des Meditationes, l'œuvre qui livrera à la modernité les conditions de la propre narration.

De toute façon, avant de nous attarder sur ce texte, nous pouvons déjà mettre en lumière à quel point la métaphore théâtrale joue un rôle tout autre que marginal dans la réflexion de Descartes. La référence du philosophe au théâtre, compendium symbolique d'une modalité renouvelée d'accès au monde, se retrouve en fait tout au long du parcours de son aventure intellectuelle: si Descartes, dans le Cogitationes Privatae (AT, X, p. 213) de jeunesse, fait du théâtre le lieu de représentation privilégié d'un complet projet de vie, dans les tardives pages des Les Passions de l'Âme il faut au contraire allusion au plaisir spontané qu'on éprouve quand, en qualité de spectateur, on assiste aux "aventures étranges qu'on voit

1 Cf. Euvres inédites de Descartes, par Foucher de Careil, Auguste Durand, Paris 1859-1860.

2 Charles Adam e Paul Tannery (AT), Euvres de Descartes, Vrin-Cnrs, Paris 1964$-1976$. 
représenter sur un théâtre" (AT, XI, p. 399). En outre, dans la troisième partie du Discours, tout en refaisant le chemin qui l'a amené à découvrir une nouvelle méthode, le philosophe dit qu'il a voyagé de par le monde, "tâchant d'y être spectateur plutôt qu'acteur en toutes les Comédies qui s'y jouent" (AT, VI, p. 28).

Le théâtre est aussi le lieu de la dissimulation, l'espace où chaque personnage peut, en endossant un masque, cacher ses véritables intentions et, de cette façon, feindre en se réfugiant dans les plis obscurs de la représentation: "Cum enim Voetius in me non prodeat nisi personatus et nunc emittat unum ex suis discipulis, nunc alium, et quae illi scripserint praestare non teneatur, ac interim tamen sua authoritate illa confirmet apud eos" (AT, VIII, p. 7). Il est par conséquent naturel que le masque du théologien réformé finisse par trouver dans le théâtre le lieu le plus adapté à la mise en scène du propre drame:

Conciones igitur istius Doctoris, modo a me descripti, populo solent placere; quotiamo ea est omnium hominum natura, ut non modo ad hilaritatem, sed maxime etiam ad quoslibet tristes animi affectus commoveri delectentur: hinc tragoediae non minus quam comediae in teatris locum inveniunt (AT, VIII, pp. 47-48).

Les diverses citations du corpus cartésien à peine rapportées ne devraient pas surprendre, surtout si on considère l'importance qu'a eue le théâtre au cœur de l'éducation jésuitique ${ }^{3}$. A ce propos, pour se faire une représentation précise de l'incidence de la métaphore théâtrale dans le modus philosophandi de Descartes, il est utile de s'attarder sur les VII Objectiones - rédigées justement par un jésuite, le théologien français Pierre Bourdin. La confrontation prend d'emblée l'aspect d'une pièce de théâtre. D'un côté, nous avons le dialogue mis en scène par le religieux, à vrai dire un monologue à plusieurs voix où l'auteur des Meditationes est une présence encombrante mais silencieuse; de l'autre côté, se fait entendre par contre Descartes, qui accuse Bourdin de l'avoir contraint à endosser un masque [larva], dans l'intention non pas de cacher, mais de déformer [non tegere, sed deformare] son visage (AT, VII, p. 454).

Après avoir démontré à plusieurs reprises les erreurs de l'adversaire, le philosophe décide de ne plus entremêler les réflexions de celui-ci avec les siennes propres, ajoutant une prémisse particulièrement intéressante:

3 Cf. Ratio atque Institutio Studiorum Societatis Iesu (1599); voir également M. Fumaroli, Les jésuites et la pédagogie de la parole, dans I gesuiti e i primordi del teatro barocco in Europa (par M. Chiabò e F. Doglio), Torre d'Orfeo, Roma 1995, pp. 39-56. 
Taedet autem me toties ipsum falsitatis arguere; dissimulabo deinceps, et reliquos ejus ludos, ad finem usque, cum silentio spectabo. Quanquam sine dispudeat videre P. R., prae nimia cavillandi cupidi tate, comicum soccum induisse; atque hic, seipsum ut meticulosum, lentum, parvi ingenii, descrivendo, non Epidicos aut Parmenones veteris Comoediae, sed hodiernae vilissimam illam personam, quae insulsi tate sua risum movere affectat, imitaru voluisse (AT, VII, pp. 492-493).

Aux yeux de Descartes, Pierre Bourdin est donc un comédien de peu de talent, prêt à tout pour retenir l'attention du public, fût-ce avec des artifices comiques de bas niveau. Le philosophe décide alors d'exposer en parallèle, dans une pièce en cinq actes, sa contrepartie dialectique en réponse aux objections présentées par le jésuite. Les VII Objectiones e Responsiones, considérées dans leur totalité, donnent par conséquent lieu à une représentation au carré, c'est-à-dire à une comédie qui part de la trame précédente pour mieux la subvertir. A la mise en scène de Bourdin se substitue, par superposition, la représentation cartésienne, une comédie pour seulement deux acteurs: avec, d'une part, le personnage de l'architecte, alter ego du philosophe, et d'autre part celui d'un stupide et ignorant maçon, qui imite à merveille la superficialité du religieux français.

La mise en scène ébauchée dans les VII Objectiones e Responsiones trouve justement dans celle des Meditationes les conditions de possibilité de son aboutissement. Nous devons donc penser aux Meditationes comme à un grand théâtre, occupé par une série de masques, savamment placés par Descartes dans l'espace de sa dramaturgie. Chaque masque, en individualisant ou en battant le rappel d'une prise de position historique ou théorique défendue précédemment, est porteur d'une visée stratégique, en ce sens qu'il joue un rôle précis dans le scénario métaphysique qui va être actualisé.

Avant que le Dieu Trompeur et le Malin Génie - deux des protagonistes du théâtre cartésien - n'entrent en scène en imposant une décisive accélération à la représentation, Descartes entame un travail de radical coup de balai à l'égard de toutes ces connaissances - désormais jugées apparentes ou non encore fondées - qui font partie depuis toujours du bagage intellectuel indispensable à l'acquisition du savoir. Le philosophe commence l'examen critique des modalités habituelles avec lesquelles on a toujours retenu opportun de considérer une connaissance comme certaine et non sujette à variation. Après avoir passé au crible de la dubitatio les informations issues des sens, puis les représentations dont la vraisemblance ne diffère pas de celle des rêves, Descartes trouve que seules "Arithmeticam, Geometriam, aliasque ejusmodi" résistent à l'examen critique. En effet, c'est parce qu'elles ne se soucient pas de l'existence réelle des objets 
qu'intuitionne leur vis épistémologique, que ces disciplines surmontent l'épreuve du doute, puisque "sive vigilem, sive dormiam, duo et tria simul juncta sunt quinque, qudratumque non plura habet latera quam quatuor; nec fieri posse videtur ut tam perspicuae veritates in suspicionem falsitatis incurrant" (AT, VII, p. 20). Toutefois, comme l'ajoute Descartes,

Infixa quaedam est meae menti vetus opinio, Deum esse qui potest omnia, et a quo talis, qualis existo, sum creatus. Unde autem scio illum non fecisse ut nulla plane sit terra, nullum coelum, nulla res extensa, nulla figura, nulla magnitudo, nullus locus, et tamen haec omnia non aliter quam nunc mihi videantur existere? Imo etiam, quemadmodum judico interdum alios errare circa ea quae se perfectissime scire arbitrantur, ita ego ut fallar quoties duo et tria simul addo, vel numero quadrati latera, vel si quid aliud facilius fingi potest? (AT, VII, p. 21).

La vetus opinio convoquée au centre du théâtre est en mesure d'étendre le doute à la connaissance qu'on a du monde, au point de mettre potentiellement en crise ces vérités-là qui n'avaient jamais été jusqu'ici, et de telle manière, mises en discussion. Ce Deus qui potest omnia se révèle être en réalité un Dieu tellement tout-puissant qu'il peut aussi être trompeur. L'omnipotentia dont il dispose est par conséquent capable d'entacher de fausseté n'importe quelle proposition cognitive. Cependant, après avoir introduit le masque du Dieu Trompeur, le philosophe français intervient de nouveau dans la mise en scène et, sans aucune véritable justification - il ne dispose en fait d'aucun critère propre à évaluer les conditions de possibilité de la figure à peine présentée - il conclut ainsi son discours: "At forte noluit Deus ita me decipi, dicitur enim summe bonus" (AT, VII, p. 21). Certes, "essent vero fortasse nonnulli qui tam potentem aliquem Deum mallent negare, quam res alias omnes credere esse incertas. Sed iis non repugnemus, totumque hoc de Deo demus esse fictitium" (AT, VII, p. 21).

Cette remarque restrictive intervient dans le discours avec l'évidente intention d'éliminer la présence perturbante que la figure du Dieu Trompeur pourrait avoir quant à la refondation épistémologique de la cogitatio. Descartes met donc en scène cette dramatis personae - l'existence de Dieu se trouve, en effet, à la base de l'entière comoedia - pour la mettre ensuite rigoureusement en question, au point de décider (de-caedere) de retirer à Dieu l'intention effective de tromper. La réflexion cartésienne fait donc appel au divin en tant que condition ultime de l'entière mise en scène; toutefois, puisque la majeure possibilité accordée à ce masque, à savoir celle de tromper, n'aurait jamais pu garantir, au-delà de tout doute raisonnable, la stabilité de l'iter cognitif que le philosophe était sur le point d'entreprendre, il devenait nécessaire d'en déformer les contours, 
d'en modifier les traits, de manière à remédier $a b$ origine à la virtualité scandaleuse qu'il a incarnée sur les planches du théâtre, même si ce n'est que furtivement.

Ce n'est donc pas un hasard si Descartes, après avoir écarté la nature du Dieu Trompeur - en excluant, sans aucune justification logique, son intention effective de tromper - prend alors personnellement position sur scène et transmet son propre rythme à l'entière narration:

Quapropter, ut opinor, non male agam, si, voluntate plane in contrarium versa, me ipsum fallam, illasque aliquandiu omnimo falsas imaginariasque esse fingam (AT, VII, p. 22).

Une nouvelle figure fait son entrée sur le théâtre: il est en réalité nécessaire de supposer "non optimum Deum, fontem veritatis, sed genium aliquem malignum", en imaginant en outre qu'un tel personnage, "summe potentem et callidum, omnem suam industriam in eo posuisse, ut me fallere" (AT, VII, p. 22).

Il est facile de relever ce qui différencie ces deux dramatis personae: tandis que le Dieu Trompeur qui intervient dans la narration s'impose à Descartes selon les contours bien établis de la vetus opinio, tout en offrant la possibilité de bouleverser l'interprétation traditionnelle, en revanche le Malin Génie, dont la figure se confond avec celles des démons qui peuplent les théâtres de l'époque baroque ${ }^{4}$, est le résultat d'un choix délibéré, et le masque qu'il affiche est parfaitement adapté à la démarche de la dubitatio cartésienne ${ }^{5}$.

La Meditatio I se conclut. Nous sommes ainsi comme les simples spectateurs d'un drame qui s'est joué ailleurs, témoins d'une dialectique serrée entre deux masqués mis en scène par un habile dramaturge. D'une part le grand Trompeur, un Dieu tellement puissant qu'il n'est soumis à aucune limitation métaphysique, logique ou éthique; d'autre part une créature médiane, un Malin Génie privé de tout pouvoir créateur, un être de fictio, dont les diverses interventions sont coordonnées par celui qui en a dessiné le masque ${ }^{6}$.

${ }^{4}$ Cf. J. Rousset, La littérature de l'âge baroque en France: Circé et le Paon, Paris 1953.

5 Sur la différence méthodologique et conceptuelle de ces deux masques, cf. H. Gouhier, Essais, Vrin, Paris 1949, pp. 143-175 et Id., La pensée métaphysique de Descartes, Vrin, Paris 1962 (n. éd. 1999), pp. 113-121; voir également F. Alquié, La découverte métaphysique de l'homme chez Descartes, Puf, Paris 1950 (n. éd. 1987), pp. 161-179.

6 Sur la finitude du Malin Génie, cf. R. Kennington, The finitude of Descartes' Evil Genius, Journal of the History of Ideas, XXXII (1971), pp. 441-446. 
Comme 1'a justement souligné Tullio Gregory ${ }^{7}$, le Dieu Trompeur et le Malin Génie, loin d'être deux figures interchangeables de l'univers médiéval dont aurait hérité Descartes, sont deux figures significativement distinctes, qui font appel à des traditions doctrinales non équivalentes. Pour la mentalité médiévale, le Malin Génie est une entité réelle 8 : l'idée qu'un démon puisse exercer un rôle dans la vie des hommes, voire même en bouleverser le cours, pouvait donc trouver confirmation dans la réalité. Ces démons, cependant, puisqu'ils n'ont qu'un statut médian dans la hiérarchie des êtres, ne sont pas du tout créateurs, mais se bornent à agir seulement sur nos représentations du monde sensible ${ }^{9}$. Le Dieu Trompeur est au contraire en mesure de susciter une précarité épistémique d'une tout autre portée. Dans un monde gouverné par un Dieu tout-puissant, libre anche de pouvoir éventuellement mentir, il ne semble pas, de fait, qu'il puisse encore y avoir pour l'homme une quelconque garantie de certitude.

Toutefois, au sein du monde médiéval, la possibilité que Dieu induise en erreur n'était en vérité qu'une hypothèse extrême dont l'éventuelle concrétisation se voyait démentie au moment même où elle venait à être pressentie. Pour la grande majorité de ces intellectuels, la potentia Dei était inséparable des paradigmes moraux avec lesquels la divine essentia confiait à l'homme les clés de son mystère. Même s'il fut toujours permis au viator de formuler d'innombrables conjectures sur l'agir ad extra de Dieu, cependant, en vue de ne pas réduire a priori l'ampleur du spectre de

7 Cf. T. Gregory, Dio Ingannatore e Genio maligno, Giornale critico della filosofia italiana, 53 (1974), pp. 477-516, maintenant dans Mundana Sapientia. Forme di conoscenza nella cultura medievale, Edizioni Storia e Letteratura, Roma 1992, pp. 401-440; trad. fr. dans le recueil Genèse de la raison classique de Charron à Descartes, Puf, Paris 2000, pp. 293-347.

8 A ce propos, il suffit de rappeler, à titre d'exemple, la figure d'Urbain Grandier, accusé d'avoir ensorcelé un couvent d'Ursulines. Cf. v. Grandier (Urbain) dans P. Bayle, Dictionnaire historique et critique (cinquième édition, IV Voll.), Amsterdam, Leyde, La Haye, Utrecht 1740, pp. 589-592; voir également A. Huxley, The Devils of Loudun (1952). Richard Popkin a considéré, en surestimant sans doute l'importance des événements qui eurent comme protagoniste le moine français, que l'affaire de Loudun a pu inspirer à Descartes son hypothèse du Malin Génie; cf. R. Popkin, The History of Skepticism, University of California Press, Berkeley-Los Angeles-London 1979.

9 Ces démons possèdent donc "la possibilité d'utiliser des species existantes ou d'agir de façon différente non seulement sur les sens extérieurs mais aussi directement sur l'imagination de façon à faire apparaître ce qui n'est pas", T. Gregory, La tromperie divine, Studi medievali, 23 (1982), pp. 517-527, maintenant dans Mundana Sapientia, Forme di conoscenza nella cultura medievale, cit., pp. 389-399, cit. p. 391. Cf., par exemple, Thomas d'Aquin, In II Sent., dist. 8, q. 1, a. 5; Sum. Theol., I, q. 111, a. 1-4; De malo, q. 6, a. 9-12; De Potentia, q. 6, a. 4-8. 
la voluntas Dei, aucune quaestio n'aurait jamais sous-entendu la présence, au plus intime de Dieu, de la moindre intention de tromper - d'ailleurs, l'actualisation éventuelle de celle-ci n'aurait été possible que si elle n'avait comporté aucune malice intrinsèque.

Pour étayer ce jugement, il suffit de s'attarder sur les positions défendues par Grégoire de Rimini et Gabriel Biel, les théologiens cités par Mersenne dans les II Objectiones (cf. AT, VII, pp. 124-126); même si ces penseurs étaient loin de les revendiquer en leur propre nom. Dans son Commentaire des Sentences de Pierre Lombard (cf. In I Sent., dist. 42-44,q. 2), le théologien de Rimini ${ }^{10}$ s'était limité à examiner de manière critique les réflexions de Richard Fitzralph et de Adam Wodeham ${ }^{11}$, deux de ses collègues contemporains ${ }^{12}$. Le tortor infantium excluait en fait catégoriquement une quelconque relation entre Dieu et le mensonge, puisque $a b$ origine la nature divine est inséparable d'une perfection morale immuable. Le Dieu vrai de la tradition est en conséquence complètement incapable de dire le faux cum intentione fallendi. Gabriel Biel ${ }^{13}$, plus radical que Gregorio dans son exégèse, ne met pas non plus en discussion le présupposé de base du collègue de Rimini. Même pour le théologien allemand, Dieu ne peut exercer son absolue causalité qu'à l'égard de ces êtres qui sont insensibles à la déception de type moral, étant don-

10 Cf. G. Leff, Gregory of Rimini. Tradition and Innovation in Fourteenth Century Thought, Manchester University Press, Manchester 1961. D'autres observations intéressantes sur les relations qui existent entre le penseur de Rimini et Descartes se trouvent dans la v. Rimini (Grégoire de), Dictionnaire historique et critique, cit., pp. 56-58.

11 Cf. G. Leff, Bradwardine and the Pelagians. A Study of His 'De Causa Dei' and Its Opponents, Cambridge University Press, Cambridge 1957; Id., Richard Fitzralph commentator of the Sentences, Manchester University Press, Manchester 1963; W. J. Courtenay, Adam Wodeham. An Introduction to His Life and Writings, Brill Academic Pub., Leiden 1978.

12 Il est important de préciser, de toute façon, que les auteurs cités et critiqués par Gregorio n'ont pas fait preuve de la radicalité dont ils sont accusés; Adam Wodeham et Richard Fitzralph ont en fait toujours situé l'éventuelle tromperie divine dans un contexte bien délimité: Dieu pourrait fallere e decipere seulement si une telle éventualité n'impliquait pas la disparition de cet ordo moral inséparable de l'essentia divine, grâce auquel Dieu administre le monde. Au cas où la tromperie serait par contre accompagnée de la connotation immorale qui la caractérise habituellement, c'est-à-dire si elle s'avérait un acte en soi injuste et pernicieux, même dans ce cas l'omnipotentia Dei ne pourrait accomplir une telle action.

13 Cf. H. A. Oberman, The Harvest of Medieval Theology. Gabriel Biel and Late Medieval Nominalism, Harvard University Press, Cambridge Mass. 1963 (n. ed. 2001), pp. 90-111; voir également Id., Masters of the Reformation, Cambridge University Press, Cambridge 1981. 
né que la deformitas et la malitia n'expriment pas une pleine réalité, mais exclusivement une privatio iustitiae debitae (cf. In II Sent., dist. 37, q. un.). Voici donc la potentia Dei libre de réaliser quoi que ce soit, pourvu, évidemment, que l'actualisation de telles possibilités ait lieu sine deformitate. Le règne de la toute-puissance divine, loin d'être purement absolutus, est depuis toujours solidaire de la dimension morale qui en est l'épiphanie. Dieu est alors en mesure de revelare falsum uniquement si une telle action n'est pas en contradiction avec le noyau le plus profond de Son essentia. Le théologien n'exclut donc pas, au moins en principe, que "Deus potest falsum aliquod revelare alicui" (cf. In III Sent., dist. 12 , q. un.), peut-être en imposant une fausse appréhension cognitive à l'intelligence humain; toutefois, une telle possibilité extrême n'est réalisable que si elle n'implique aucun peccatum ni aucune malitia destinée à tromper moralement. Au sein du monde médiéval, l'indiscutable moralité de Dieu servait donc de rempart sûr contre la prolifération des ces quaestiones.

Le Dieu Trompeur du théâtre cartésien est néanmoins de nature vraiment singulière. Ses conditions de possibilité et le contexte conceptuel d'où il tire son essentia s'appuient sur un paradigme métaphysique tout à fait différent de celui qui avait guidé jusqu'alors les analyses médiévales. C'est proprement à cause de sa théorie de la liberté qu'a Dieu de créer les vérités éternelles ${ }^{14}$ que Descartes est amené à mettre en scène le masque d'un Dieu trompeur ${ }^{15}$. En effet, avant que le philosophe français n'introduise dans l'horizon de l'époque la libre création des vérités éternelles, celles-ci, tant logiques et mathématiques que morales, étaient directement relatives à la cogitatio Dei, sans aucune restriction. On ne voyait par conséquent pas de contradiction entre leur caractère éternel et la dépendance où elles se trouvaient par rapport à la perfection et à

14 La théorie sur les vérités éternelles apparaît en divers endroits de la réflexion cartésienne: cf. AT, I, pp. 145-146; AT, I, pp. 149-150; AT, I, pp. 151-153; AT, II, p. 138; AT, IV, pp. 118-119; AT, V, pp. 223-224; AT, V, p. 272; AT, VII, p. 380; AT, VII, pp. 431-43; AT, VII, pp. 435-436.

15 Cf. E. Bréhier, La création des vérités éternelles dans le système de Descartes, Revue philosophique de la France et de l'Etranger, 62 (1937), pp. 15-29; H. Gouhier, La pensée métaphysique de Descartes, cit., pp. 250-264; J-L. Marion, Sur la théologie blanche de Descartes, Puf, Paris 1981, pp. 328-346. Pour une analyse critique sur la possibilité de voir à l'œuvre une telle doctrine, cf. E. Scribano, Angeli e beati. Modelli di conoscenza da Tommaso a Spinoza, Laterza, Roma-Bari 2006, pp. 161-194. Sur l'importance d'une telle théorie dans le panorama philosophique de l'époque, cf. G. Gasparri, Le Grand Paradoxe de M. Descartes. La teoria cartesiana delle verità eterne nell'Europa del XVII secolo, Leo S. Olschki, Roma 2007. 
l'infinité de Dieu qui les conçoit. L'objectivité qui caractérise l'essence universelle des choses était alors connue éternellement de Dieu, tout en étant concrètement distincte de lui. De semblables vérités étaient ainsi nécessaires au point de se trouver ab origine dans l'intellect divin, comme si elles y étaient des modèles exemplaires d'emblée inséparables de sa cogitatio et auxquels la voluntas divine s'était conformée pour donner corps à sa propre creatio.

A partir de ces prémisses, on aperçoit clairement la raison pour laquelle on n'admettait pas dans la précédente tradition, en dépit des quaestiones avancées, qu'un Dieu tout-puissant puisse tromper. La possibilité de la tromperie divine était en fait en évidente contradiction avec la nature morale de Dieu, à savoir avec l'un des caractères infinis de sa divine et éternelle essentia. Le système de ses attributs est en réalité consubstantiel à l'infinie possibilité de ses diverses théophanies au point de servir de repère précis au viator, pour s'orienter dans les arcanes de la cogitatio Dei. Si Dieu est bon autant qu'il est tout-puissant - l'infinie potentia divine ne peut certes se contredire -, on doit alors exclure qu'un Dieu à la fois infiniment bon et tout-puissant prenne la liberté, en vertu de son omnipotentia, de nous tromper.

La vraie rupture cartésienne se marquera justement dans le fait de soutenir qu'une telle exclusion soit librement voulue par Dieu. S'adressant à Mersenne, Descartes justifie en ces termes sa conviction:

Les vérités mathématiques, lesquelles vous nommez éternelles, ont été établies de Dieu et en dépendent entièrement, aussi bien que tout le reste des créatures. C'est en effet parler de Dieu comme d'un Jupiter ou Saturne, et l'assujettir au Styx et aux Destinées, que de dire que ces vérités sont indépendantes de lui. Ne craignez point, je vous prie, d'assurer et de publier partout, que c'est Dieu qui à établi ces lois en la nature, ainsi qu'un Roi établit des lois en son Royaume (AT, I, p. 145).

Non seulement les vérités éternelles dépendent totalement de Dieu, devant à sa seule voluntas leurs raisons d'être, mais plus radicalement, comme l'écrit Descartes dans une lettre ultérieure, ces vérités n'ont aucune nécessité intrinsèque qui n'ait été $a b$ origine établie par Dieu:

Pour les vérités éternelles, je dis derechef que sunt tantum verae aut possibiles quia Deus illas veras aut possibiles conosci, non autem contra veras a Deo cognosci quasi independenter ab illo sint verae (AT, I, p. 149). 
La causalité divine se révèle absolue et il n'y a donc pas de modèles passibles de conditionner le libre exercice de la voluntas Dei. En dépit des interprétations de Martial Guéroult ${ }^{16}$ et de Edwin Curley ${ }^{17}$, il n'existe par conséquent aucun paradigme éternel, même généré par l'essentia Dei, qui ne soit dû à l'action de sa puissance. D'ailleurs, puisqu'il représente la ratio de tout bien et de toute vérité concevables ${ }^{18}$, Dieu est exempt d'un quelconque lien de dépendance, son omnipotentia étant en définitive la condition de toute hypothétique limitation. L'homme n'est alors autorisé à rien exclure du champ de ce que Dieu est en mesure de réaliser: la raison humaine ne peut en fait juger, à cause des limites inhérentes à sa connaissance, de l'ampleur infinie de la liberté divine ${ }^{19}$, si ce n'est en imposant à Dieu les critères propres à sa condition d'être créé 20 .

Surtout, outre à être le fruit d'une libre décision divine et en rapport immédiat de dépendance avec elle, les vérités éternelles sont, en dernière analyse, complètement contingentes:

Vous demandez aussi qui à nécessité Dieu à créer ces vérités: et je dis qu'il a été aussi libre de faire qu'il ne fût pas vrai que toutes les lignes tirées du centre à la circonférence fussent égales comme de ne pas créer le monde. Et il est certain que ces vérités ne sont pas plus nécessairement conjointes à son essence, que les autres créatures. Vous demandez ce que Dieu a fait pour les produire. Je dis que ex hoc ipso quod illas ab aeterno esse voluerit et intellexerit, illas creavit, ou bien (si vous n'attribuez le mot de creavit qu'ò l'existence des choses) illas disposuit et fecit. Car c'est en Dieu une même chose de vouloir, d'entendre, et de créer, sans que l'un précède l'autre, ne quidem ratione (AT, I, pp. 151-152).

16 Cf. M. Guéroult, Descartes selon l'ordre des raisons (L'âme et les corps, Vol. II), Aubier, Paris 1953, pp. 22-39.

17 Cf. E. M. Curley, Descartes on the Creation of the Eternal Truths, The Philosophical Review, 4, XCIII (1984), pp. 569-597; voir également P. T. Geach, Omnipotence, Philosophy, XLVIII (1973), pp. 7-20.

18 Cf. AT, VII, pp. 435-436; AT, V, pp. 223-224.

19 Cf. AT, IV, pp. 118-119; AT, V, pp. 223-224; AT, V, p. 272.

20 Il n'est donc pas permis, en suivant les suggestions de Amos Funkenstein et Hide Ishiguro, de nier que Dieu soit impuissant face à la contradiction, en arguant que notre episteme est nécessairement uniforme aux préceptes du principium firmissimum. Cf. A. Funkenstein, Theology and the Scientific Imagination from the Middle Ages to the Seventeenth Century, Princeton University Press 1986; Id., Descartes, Eternal Truths and the Divine Omnipotence, dans Studies in the History and Philosophy of Science, Vol. VI, n. 3, Pergamon Press 1975, pp. 185-199. Cf. H. Ishiguro, The status of necessity and impossibility in Descartes, dans A. O. Rorty (ed.), Essays on Descartes' Meditations, University of California Press, Berkeley 1986, pp. 459-472. 
La contingence des vérités éternelles est mise en relation directe avec l'affirmation d'une toute-puissance proprement inédite. A vrai dire, indifférent à la totalité des possibles, Dieu aurait tout aussi bien pu, s'il l'avait trouvé opportun, structurer le monde selon des proportions logiques, mathématiques et morales tout à fait différentes. Une telle conception de sa potentia introduit donc une discontinuité absolue: comme l'a justement souligné Harry Frankfurt ${ }^{21}$, l'interprétation cartésienne amène à un hiatus insurmontable entre la raison humaine et la révélation divine, écartant d'emblée la possibilité d'une quelconque univocité conceptuelle entre elles. La théorie de la nature créée des vérités éternelles est par conséquent ce qui justifie qu'un masque comme celui du grand trompeur puisse apparaître sur scène, fût-ce le temps requis pour tracer les limites de la représentation ${ }^{22}$. Mais ce personnage, qui fournit, uno actu, et le mobile du drama et la possibilité de son rapide dénouement, devra être sans délai démasqué, pour redonner du même coup mouvement et jeu aux autres intervenants dans la pièce. Aussitôt après avoir relégué dans les coulisses celui qui porte le masque du trompeur, Descartes appellera à la rescousse d'autres acteurs et d'autres figures plus adaptés à une heureuse conclusion de la comédie. Après qu'il aura donné son congé au Dieu Trompeur et tiré parti au mieux du modeste malin démon, il aura recours, au terme d'un long et fatigant parcours, à la figure du Dieu Vrai qui seul pourra fournir au philosophe de quoi mettre fin à sa dubitatio.

Maintenant que nous avons étudié le théâtre des Meditationes et décrit le rôle joué par les divers masques qui y sont à l'œuvre, nous pouvons revenir sur les débuts de la spéculation de Descartes, en nous attardant en particulier sur ce Traité de la Lumière qu'il ne publia jamais, suite à la condamnation infligée alors à Galilée ${ }^{23}$. Il est important de noter que Descartes entreprend la rédaction de ce traité au moment où il conçoit sa

${ }^{21}$ Cf. H. Frankfurt, Descartes on the Creation of the Eternal Truths, The Philosophical Review, 1, LXXXV (1977), pp. 36-57. Voir également L. Alanen, Omnipotence, Modality and Conceivability, dans J. Broughton and J. Carriero (ed.), A Companion to Descartes, Blackwell Publishing Company 2008, pp. 353-371; Id., Descartes, Omnipotence and Kinds of Modality (1988), dans V. Chappell (ed.), Essays on Early Modern Philosophers, Vol. I: René Descartes, Garland Publishing, New York \& London 1992, pp. 182-196.

22 Le Dieu Trompeur, naturellement, n'est pas, sic et simpliciter, le Dieu créateur des vérités éternelles décrit dans la correspondance cartésienne, même s'il y laisse de toute façon une trace. D'ailleurs, c'est uniquement si on fait implicitement référence à un Dieu qui crée librement - il est en effet la ratio de tout bien ou de toute vérité possibles et le garant des paradigmes et des modèles de tout ce qui existe - qu'il est aussi possible de dessiner les traits de cette dramatis personae.

23 Cf. AT, I, pp. 270-272. 
doctrine sur la nature créée des vérités éternelles. Ce n'est en effet pas un hasard s'il fait, dans sa lettre du 15 avril 1630 adressée à Mersenne, explicitement allusion au rapport étroit qui existe entre sa novitas métaphysique et son projet d'une physique intégrale:

J'espère écrire ceci, même avant qu'il soit 15 jours, dans ma physique; mais je ne vous prie point pour cela de le tenir secret; au contraire je vous convie de le dire aussi souvent que l'occasion s'en présentera, pourvu que ce soit sans me nommer (AT, I, p. 146).

Cet extrait, où sont manifestes les précautions que prend Descartes dans sa correspondance philosophique, suggère en outre, comme nous le montrons ci-après, la présence d'une dialectique latente dans l'exposé de sa réflexion. Pour mieux souligner la parfaite continuité qu'il y a entre sa physique et sa théorie de la nature créée des vérités éternelles, Descartes imagine en effet le dialogue suivant:

On vous dira que si Dieu avait établi ces vérités, il les pourrait changer comme un Roi fait ses lois; à quoi il faut répondre que oui, si sa volonté peut changer. - Mais je les comprends come éternelles et immuables. - Et moi je juge le même de Dieu. - Mais sa volonté est libre. - Oui, mais sa puissance est incompréhensible; et généralement nous pouvons bien assurer que Dieu peut faire tout ce que nous pouvons comprendre, mais non pas qu'il ne peut faire ce que nous ne pouvons pas comprendre; car ce serait témérité de penser que notre imagination a autant d'étendue que sa puissance (AT, I, pp. 145-146).

L'accent mis sur l'incompréhensibilité de la potentia Dei nous indique la direction à suivre. L'homme, en réalité, à cause des limites de son entendement, ne dispose d'aucun critère décisif pour s'orienter in mente Dei: il ne peut juger du bien fondé des décisions divines qu'en fonction de son humaine raison. L'immuabilité et la nécessité que nous prêtons aux vérités éternelles sont alors destinées à pouvoir signifier peu de chose quand on les replace dans le contexte d'une potentia absolue et indifférente. Nous ne pouvons par conséquent pas exclure, confinés comme nous le sommes dans un espace tout à fait limité et héritiers d'une logique d'êtres créés, que Dieu puisse revoir le contenu de ces vérités, quitte à bouleverser nos catégories cognitives. Ce qui ne veut pas dire, naturellement, que ces vérités soient sujettes au changement; Descartes suggère plutôt, si on prend sa pensée dans son sens le plus radical, que l'homme ne peut refuser l'éventualité de leur possible changement ${ }^{24}$ sans 
affaiblir cette omnipotentia incompréhensible qui représente, comme l'a montré Jean-Luc Marion, le nom cartésien de Dieu ${ }^{25}$.

Quoi qu'il en soit de leur pertinence, ces considérations n'interdisent pas que l'episteme humaine puisse acquérir un savoir qui soit nécessaire; elles laissent entrevoir, par contre, une possible réponse à la question que soulève la réflexion de Descartes: puisqu'il n'exclut pas la possibilité que la liberté d'action de Dieu échappe aux catégories de la ratio humaine, il ne peut non plus exclure que l'objectivité inhérente à notre appareil

24 Il existe pourtant un témoignage recueilli par Frans Burman où Descartes semble fournir une réponse en partiel désaccord avec notre lecture: "Seda an ergo potuisset Deus imperare Creaturae ut se odisset, et hoc bonum ita instituisse? Rsp. Jam non potest, sed quid potuerit nescimus; et quidni creaturae hoc imperare potuisset?" (AT, V, p. 160). Dieu, suggère le philosophe, aurait sûrement pu modifier $a b$ origine les lois morales en vigueur, en ordonnant à une de ses créatures de le haïr; dans l'état actuel des choses, il ne semble cependant pas qu'une semblable éventualité soit encore réalisable. Il importe d'ailleurs de souligner que le Entretien avec Burman, bien que faisant partie du corpus cartésien, n'est pas de la main de Descartes, mais est seulement le compte rendu par le jeune Burman, étudiant en théologie et fils d'un responsable protestant, de son colloque avec le philosophe français. En outre, il y a divergence de dates entre le moment de la rencontre - 16 avril 1648 - et celui de son compte rendu écrit - 20 avril 1648 (il faut en plus rappeler que le manuscrit dont on dispose n'est pas le texte original du compte rendu, mais seulement une copie exécutée à Amsterdam par Johan Clauberg). Cela veut dire que ce texte - issu précisément d'un colloque, en quelque sorte une interview philosophique avant la lettre - ne fut vraisemblablement pas rédigé le jour de la rencontre. Cette divergence de dates, ajoutée à la maigre formation intellectuelle de l'étudiant, nous autorise à sous-estimer l'importance qu'on peut accorder à ce témoignage. A confirmation de notre jugement, il y a, entre autres, un passage du colloque où Descartes semble défendre des positions sur la nature des vérités éternelles en complète contradiction avec les autres témoignages dont nous disposons: "Nec refert illa decreta a Deo separari potuisse, hoc enim vix dici debet, quamvis enim Deus ad omnia indifferens sit, necessario tamen ita decreti, quia necessario optimum voluit, quamvis sua voluntate id optimum fecerit, nec deberet hic sejungi necessitas et indifferentia in Dei decretis, et quamvis maxime indifferenter egerit, simul tamen maxime necessario egit" (AT, V, p. 166). Si la première partie du passage n'est guère compatible avec la pensée de Descartes, la seconde est en revanche ouvertement contradictoire, comme l'a noté Marion lui-même, Sur la théologie blanche de Descartes, cit., pp. 306-307. Puisque ce passage a été à juste titre considéré comme étranger à la pensée de Descartes (cf., par exemple, AT, VII, pp. 431-432; AT, VII, pp. 435-436), il est sans doute opportun de ne pas voir dans le colloque un témoignage suffisamment fiable pour fonder une interprétation. Il est en effet probable que Burman, dans sa tentative de mettre par écrit le récit de sa confrontation avec Descartes, ait commis quelque maladresse d'interprétation, parce qu'il ne disposait pas des instruments suffisants pour apprécier en profondeur le sens d'une théorie aussi complexe et détaillée.

25 Cf. J.-L. Marion, Sur la théologie blanche de Descartes, cit., p. 281. 
cognitif puisse être modifiée de par une décision divine souveraine, même si elle nous reste fondamentalement incompréhensible.

$\mathrm{Au}$ point où on en est, on s'attendrait à trouver dans le traité de Descartes une allusion au lien indissociable qu'il établit entre sa pensée métaphysique et sa physique. Nos attentes, au moins à première vue, semblent cependant déçues.

Le septième chapitre de Le Monde traite des lois qui devraient gouverner ce "nouveau monde". Deux conditions nécessaires président à leur formulation: que Dieu soit immuable (AT, XI, p. 38) ${ }^{26}$, et qu'il maintienne la nature dans l'état où il l'a créée (AT, XI, pp. 36-37). Après avoir détaillé les trois lois qui gouvernent l'ensemble des phénomènes naturels, Descartes fournit une ultérieure précision:

Je me contenterai de vous avertir, qu'outre les trois lois que j'ai expliquées, je n'en veux point supposer d'autres, que celles qui suivent infailliblement de ces vérités éternelles sur qui les Mathématiciens ont accoutumé d'appuyer leurs plus certaines et plus évidentes démonstrations; ces vérités, dis-je, suivant lesquelles Dieu même nous a enseigné qu'il avait disposé toutes choses en nombre, en poids, et en mesure; et dont la connaissance est si naturelle à nos Ames, que nous ne saurions ne les pas juger infaillibles, lorsque nous les concevons distinctement; ni douter que si Dieu avait créé plusieurs Mondes, elles ne fussent en tous aussi véritables qu'en celui-ci (AT, XI, p. 47).

Quand on confronte cette dernière remarque sur les vérités éternelles avec le statut initial de celles-ci discuté dans sa correspondance avec le Minime, on note de la part du philosophe une nette différence d'accent. D'une part, on soutient la contingence de semblables vérités, librement créées par une potentia infinie et incompréhensible; d'autre part, on défend une position différente, insérée dans un autre contexte, mais élaborée, dans la même période, comme le montrent les lettres déjà citées. En fait, dans Le Monde, le statut ontologique des vérités éternelles est nécessaire: il ne pourrait changer même au cas où Dieu créerait d'autres mondes possibles. Il existe donc un critère de proportion tel qu'il consent aux recherches de l'homme un solide ancrage à l'éternelle vérité de l'être.

${ }^{26}$ La conviction cartésienne que l'immuabilité et la constance divines soient nécessaires pour garantir une connaissance stable du monde revient aussi dans les Principia Philosophiae. La cause générale du mouvement est, du reste, "Deum ipsum, qui materiam simul cum motu et quiete in principio creavit, jamque, per solum suum concursumordinarium, tantundem motus et quietis in ea tota quantum tunc posuit conservat". Infatti, "intelligimus etiam perfectionem esse in Deo, non solum quod in se ipso sit immutabilis, sed etiam quod modo quam maxime constanti et immutabili operetur" (AT, VIII, p. 61). 
Si nous faisons un pas en arrière et prêtons suffisamment attention à la conclusion du cinquième chapitre de Le Monde, nous pouvons relever un indice capable de nous fournir quelques critères importants d'appréciation de la stratégie cartésienne. Avant d'entamer la description de ce nouveau monde, Descartes formule, à l'adresse d'un lecteur potentiel, la brève prémisse suivante:

Il me reste ici encore beaucoup d'autres choses à expliquer, et je serais même bien aise d'y ajouter quelques raisons pour rendre mes opinions plus vraisemblables: mais afin que la longueur de ce discours vous soit moins ennuyeuse, j'en veux envelopper une partie dans l'invention d'une fable au travers de laquelle j'espère que la vérité ne laissera pas de paraître suffisamment, et qu'elle ne sera pas moins agréable à voir, que si je l'exposais toute nue (AT, XI, p. 31).

Le parcours cognitif qui sera décrit se déroulera donc dans un statut ontologique de fable. Les avantages qu'on en retirera devront alors être intégrés dans un horizon typiquement affabulatoire. Etant donné que le cheminement déductif que la raison devra accomplir pour donner forme au monde est inséré dans cette fable, ce n'est pas un hasard si Descartes, avant de détailler les lois qui devront régir les mécanismes de ce monde, fournit sans tarder une précision d'importance notable: "Mon dessein n'est pas d'expliquer comme eux les choses qui sont en effet dans le vrai monde; mais seulement d'en feindre un à plaisir" (AT, XI, p. 36).

La fictio de cette fable a donc une construction délibérée, exempte d'une vérité qui n'ait pas été présupposée d'emblée. Voilà que la valeur intrinsèque de ce qui devra être démontré n'aura d'autre référent que cette décision de constituer un monde et d'en définir clairement les règles du jeu, tout en laissant délibérément dans l'ombre les conditions de sa possibilité. Les lois de ce nouveau monde décriront donc les seuls secrets de cette fable, abandonnant "les choses qui sont en effet dans le vrai monde" à un autre sort.

Dans l'œuvre de Descartes, la fable et le caractère théâtral de la représentation - fabula est en réalité synonyme de représentation scénique, tragique ou comique - sont souvent utilisées pour transmettre une décisive accélération au raisonnement en cours, comme s'ils constituaient un espace privilégié pour amener le parcours déductif ou narratif à un plan supérieur. On peut voir à l'œuvre cette technique discursive dans nombre d'écrits cartésiens, qu'il s'agisse des Cogitationes citées au début de notre article ou du préambule officiel du Discours, sans oublier La recherche et les Regulae. Si dans les fragments de jeunesse, le théâtre sert de scène symbolique aux futurs scénarios de toute une vie, dans le Discours de la 
Méthode, c'est la fable qui est par contre le moyen privilégié pour exposer progressivement une méthode - "Ne proposant cet écrit que comme une histoire, ou, si vous l'aimez mieux, que comme une fable" -, puisqu'on sait à quel point "la gentillesse des fables" sait favoriser "l'esprit" (AT, VI, pp. 4-5).

La même expression apparaît aussi dans un passage décisif des Regulae ad Directionem Ingenii, c'est-à-dire dans cette douzième règle qui, synthétisant le développement précédent, décrit les conditions qui permettent à la pensée d'arriver à un savoir exempt d'erreur. L'entendement, en réalité, "a nullo unquam esperimento decipi posse, si praecise tantum intueatur rem sibi objectam", à condition donc de ne pas se fier à l'imagination pour juger de la vérité des sensations, ni aux sensations pour se représenter les réalités externes ou se persuader que celles-ci existent réellement comme elles nous apparaissent d'habitude. En vérité, l'imbrication de l'imagination, de la sensation et du monde externe expose l'homme à l'éventualité de l'erreur, "ut si quis fabulam nobis narraverit, et rem gestam esse credamus" (AT, X, p. 423).

La fabula se révèle ici en rapport immédiat avec le piège, celle-là précisément qui mine radicalement l'acquisition d'une connaissance universellement garantie. Ce leitmotiv de la fable est également présent dans La Recherche de la Vérité, un écrit où l'instance théâtrale, comme lieu privilégié du déploiement de la déduction philosophique, est nouvellement à l'œuvre. On peut voir dans un passage décisif que la pensée cartésienne doit sa progression au déroulement des plis de son arrière-plan baroque et d'une métaphore théâtrale:

N'avez-vous jamais ouï ce mot d'étonnement dedans les comédies: Veillé-je, ou si je dors? Comment pouvez-vous être certain que votre vie n'est pas un songe perpétuel, et que tout ce que vous pensez apprendre par vos sens n'est pas faux, aussi bien maintenant comme lorsque vous dormez? Vu principalement que vous avez appris que vous étiez créé par un être supérieur, lequel étant tout-puissant, comme il est, n'aurait pas eu plus de difficulté à nous créer tel que je dis, que tel que vous pensez que vous vous êtes (AT, X, pp. 511-512).

Cette dernière citation montre que la fragilité épistémique provient autant de la mystification d'un être supérieur qui endosse le masque du Dieu Trompeur, notamment dès le début des Meditationes, que de l'usage dramaturgique et ontologique de la fabula par Descartes tout au long de sa 'publique' réflexion philosophique.

Mais en insistant sur l'importante utilisation des vertus du théâtre et de la fable dans la spéculation cartésienne, nous avons laissé dans l'ombre 
une dynamique bien présente dans son œuvre, qu'il importe désormais d'éclairer de plus près. Dans Le Monde, les réflexions qui concernent le statut ontologique des vérités éternelles sont en fait bien différentes de celles par lesquelles Descartes, dans ses lettres de 1630, fait dépendre ces mêmes vérités du bon plaisir de la voluntas Dei. Cette différence d'interprétation révèle la présence d'une dialectique à l'intérieur de la pensée cartésienne. Cependant, comme ces deux définitions de la nature des vérités éternelles datent de la même période et comme il n'est pas permis de penser que Descartes se contredise ou veuille élaborer deux métaphysiques incompatibles entre elles, il ne nous reste qu' à nous interroger sur les raisons qui l'ont poussé à une double formulation d'un même problème.

La fin du sixième chapitre du Traité sur la Lumière offre un élément de réponse. Avant que l'immuabilité de Dieu ne donne aux lois leur fondement stable et avant que les vérités éternelles ne soient jugées nécessaires en dépit de l'infinie potentia Dei, Descartes, qui s'est autorisé à inventer un monde à plaisir, y fournit une importante précision méthodologique:

Si j'y mettais la moindre chose qui fût obscure, il se pourrait faire que parmi cette obscurité il y aurait quelque répugnance cachée dont je ne me serais pas aperçu, et ainsi que sans y penser je supposerais une chose impossible; au lieu que pouvant distinctement imaginer tout ce que j'y mets, il est certain qu'encore qu'il n'y eût rien de tel dans l'ancien monde, Dieu le peut toutefois créer dans un nouveau: car il est certain qu'il peut créer toutes les choses que nous pouvons imaginer (AT, XI, p. 36).

C'est sur ces derniers mots que se termine le sixième chapitre. Après avoir éliminé toute obscurité, le philosophe peut se frayer un chemin dans son nouveau monde ${ }^{27}$, convaincu de pouvoir décrire en toute vérité les moindres détails de la fable. La citation qu'on vient de reproduire semble suggérer que si l'auteur de cette fabula n'intervenait pas délibérément dans la narration, de manière à lui réserver une heureuse issue, il pourrait exister quelque contenu ou chose obscure capable de bouleverser tout le récit. Pour écarter ce danger, il est alors nécessaire que la clarté et la distinction des contenus de la fable soient abstraits, c'est-à-dire éloignés et séparés de quoi que ce soit qui puisse embrouiller la représentation

27 Dans sa correspondance, Descartes parle souvent, en relation avec le traité de physique qu'il est en train de concevoir, de la description de 'son' monde: "Je ne pense pas après ceci me résoudre jamais plus de faire rien imprimer, au moins moi vivant: car la fable de mon Monde me plaît trop pour manquer à la parachever" (AT, I, p. 179). 
qui est en train de se dérouler. L'obscurité qui pourrait s'installer sur le théâtre est en fait une réalité qui, même si elle n'est pas d'emblée due aux limites de l'entendement humain, est libre de s'introduire dans ses marges. Loin d'être l'objet d'une proportion, elle est ainsi ce qui en définit la possibilité. Il s'agit, en somme, de la volonté de Dieu considérée dans sa toute-puissance. Voilà ce que Descartes est en train de sous-entendre dans la citation: si nous ne voulions rendre univoque le pouvoir divin, à savoir si nous ne décidions de ramener l'omnipotentia Dei à la dimension de notre capacité de compréhension - de manière à définir et limiter a priori l'espace conceptuel qui dérive de la nature créée des vérités éternelles nous ne pourrions rendre raison du monde dont nous faisons l'expérience. Et nous ne pourrions le comprendre de manière nécessaire, puisque nos propres modalités cognitives pourraient se révéler, à la lumière d'une telle logique métaphysique, purement contingentes. Toutefois, parce que nous devons arriver au plein aboutissement de nos efforts intellectuels, de manière à acquérir les instruments qui peuvent nous garantir une représentation stable et fonctionnelle du monde que nous habitons, il est opportun que l'extension de la puissance divine soit proportionnée aux limites de notre entendement. Il est par conséquent nécessaire de penser la toute-puissance de Dieu comme étant depuis toujours proportionnée à des paradigmes métaphysiques et mathématiques indépendants de tout changement.

D'ailleurs, la description du nouveau monde n'aurait jamais pu être réalisée, ainsi qu'elle l'a été, si la théorie cartésienne sur les vérités éternelles n'avait été exposée à part du Traité. C'est la raison pour laquelle la métaphysique cartésienne, reconnue dans toute sa radicalité, condamne le monde externe à n'être qu'une fabula ${ }^{28}$, à savoir une fictio qui, tout en ayant des lois qui président à la cohérence de son développement, ne possède pas une vérité propre qui soit en mesure de le justifier au-delà d'un doute raisonnable, c'est-à-dire en dépit de la libre autant que toute-puissante volonté de Dieu. Il est alors indispensable d'éliminer cette chose obscure en vue de conclure le récit sans qu'aucune ombre ne vienne réduire la clarté et la distinction qui devront dominer l'entière narration. Après avoir expulsé de la scène du monde la nature ombreuse du divin,

28 Comme le souligne justement Jean-Pierre Cavaillé, quand il établit une étroite relation entre le choix stratégique de Le Monde et la théorie de la libre création des vérités éternelles, "la métaphysique est cependant effectivement présent dans le Monde et cette présence seule justifie pleinement le recours à la fable (...) La métaphysique de la libre création, qui concerne de la même façon les essences et les existences, condamne à la fois le monde absolue, qu'un songe, une fable", J.-P. Cavaillé, Descartes. La fable du monde, Vrin, Paris 1991, p. 243. 
Descartes pourra alors se frayer un chemin in mente Dei, en concevant sa nature divine selon ces caractéristiques qui sont nécessaires à l'heureuse conclusion de la fable

La stratégie de Descartes est donc en train de s'exercer dans cette œuvre de dé-cision de la toute-puissance divine qui trouve son apogée dans le théâtre des Meditationes. C'est là que le masque du Dieu Trompeur a fait son entrée, mais a été aussitôt retiré, pour préserver l'entière comédie de la précarité qui aurait pu la menacer. De façon analogue, dans le passage décisif de Le Monde, avant la description des lois de nature destinées à le gouverner, l'ébauche de l'entière physique est portée en scène pour être immédiatement exorcisée, seulement éclairée par cette lumière qui ne pourra permettre aucun doute dans le parcours de reconstruction du savoir.

Les conclusions de notre analyse confirment la complexité que recèle la réflexion cartésienne au point de suggérer l'existence d'une véritable dialectique entre un Descartes 'public' et un D'escartes 'privé' ${ }^{29}$. Il semble donc y avoir, comme l'a souligné Emanuela Scribano ${ }^{30}$, une dénivellation entre la 'métaphysique publique' du philosophe - un discours qui choisit de ne pas aborder explicitement la théorie sur la création des vérités éternelles - et sa pensée philosophique la plus profonde dissimulée, en particulier, dans sa correspondance ${ }^{31}$.

Toutefois, ces dernières considérations sur la philosophie de Descartes ne prétendent pas suggérer l'existence chez lui de deux réflexions antagonistes, comme si nous nous trouvions en présence de deux niveaux de pensée parallèles et inconciliables. Elles visent au contraire à

29 Nous sommes en train de faire référence au jeu de mots repéré dans une lettre envoyée à Descartes, où Huygens fait allusion à la tendance qu'a le philosophe français de vivre à l'écart, loin du commerce de la société et des contraintes qu'elle comporte (cf. AT, III, p. 745).

30 Cf. E. Scribano, Angeli e beati. Modelli di conoscenza da Tommaso a Spinoza, cit., pp. 184-189.

31 Pour autant, nous n'avançons pas l'hypothèse d'un Descartes 'ésotérique', qui aurait l'intention de soustraire une part de ses recherches à la connaissance et à la discussion publiques. Cette idée défendue en son temps par Maxime Leroy dans Descartes, le philosophe au masque (1926), à savoir celle d'un philosophe qui aurait eu l'intention de dissimuler les racines libertines de sa pensée, ne trouve aucune confirmation convaincante dans les textes cartésiens. D'ailleurs, comme l'a bien vu Jean-Luc Marion, c'est parce que le propre de la correspondance à l'époque classique est justement d'être un instrument indispensable à la circulation des idées, qu'il y a lieu de repousser une telle interprétation (cf. J.-L. Marion, La création des vérités éternelles. Le réseau d'une 'question', dans J.-R. Armogathe, G. Belgioioso e C. Verti, La biografia intellettuale di René Descartes attraverso la Correspondance, Vivarium, Napoli 1999, pp. 387-407). Selon Carlo Borghero, les lettres sont en fait un élément important d'une "stratégie communicative": l'auteur de celles-ci est 
mettre en évidence la complexité qui caractérise la pensée cartésienne, dans l'intention de l'examiner et peut-être d'élucider les raisons qui la sous-tendent. Puisqu'il n'est pas légitime de se référer à un tri sélectif dans l'œuvre de Descartes entre la réflexion philosophique réservée à des correspondants et celle qui est officiellement destinée à la publication, il ne nous reste qu'à retrouver dans les textes la présence de la dialectique qui anime la pensée du philosophe.

L'idée qu'il y ait quelque différence entre la pensée la plus personnelle de Descartes et celle plus académique de ses publications nous est suggérée à divers endroits du corpus cartésien. Parmi les exemples en mesure d'appuyer notre interprétation, il y a, dans la Meditatio VI, celui de l'hydropique ${ }^{32}$, cet homme poussé de par sa nature à désirer ce qui lui est nocif. La question se pose de savoir pourquoi un Dieu bon et vrai n'intervient pas pour remédier à une aussi pénible situation. En effet, Descartes considère qu'il n'y a rien dans le monde qui n'atteste de la puissance et de la bonté de Dieu [Dei potentiam bonitatemque]; en outre, il est convaincu que la volonté divine aurait pu modifier la nature de cet homme et donc supprimer les causes de ce dysfonctionnement. Cependant, s'il avait agi en ce sens, Dieu ne serait plus arrivé à conserver au corps sa condition remarquable. Il ne reste alors à Descartes qu'à reconnaitre l'existence du problème:

Ex quibus omnino manifestum est, non obstante immensa Dei bonitate, naturam hominis ut ex mente et corpore compositi non posse non aliquando esse fallacem (AT, VII, p. 88).

Dieu, bien qu'il veuille que l'hydropique ne soit pas torturé par la soif, ne semble pas en mesure de concrétiser son intention. Sa voluntas est donc impuissante face à une situation qu'elle souhaiterait éviter. Néanmoins, Descartes n'est amené à accepter cette conséquence que parce qu'il décide, en pleine connaissance de cause, de mettre entre parenthèses ce

\footnotetext{
bien conscient que le destinataire pourrait divulguer leur contenu, aussi les rédige-t-il en prévoyant cette éventualité (cf. C. Borghero, La corrispondenza, il testo, il laboratorio, dans La biografia intellettuale di René Descartes attraverso la Correspondance, cit., pp. 715-724). On peut donc penser que si Descartes a choisi d'exposer sa métaphysique sans y mentionner la nature librement créée des vérités éternelles, ce n'est pas dans l'intention d'installer une distance entre lui et les correspondants avisés de ses lettres, c'est plutôt pour éviter, de la part d'un public de non spécialistes, une malencontreuse interprétation d'une théorie aussi radicale que la sienne.

32 Cf. E. Scribano, Quel che Dio non può fare. Descartes e i limiti della potenza divina, dans G. Canziani, M. A. Granada, Y. Ch. Zarka, Potentia Dei. L'onnipotenza nel pensiero dei secoli XVI e XVII, Franco Angeli, Milano 2000, pp. 335-350.
} 
Dieu indifférent et librement créateur qu'il évoque à plusieurs reprises dans sa correspondance.

Nous relevons un autre exemple à l'appui de l'hiatus qui semble tenir sous tension la spéculation de Descartes dans un des passages de son œuvre où il s'interroge sur la possibilité qu'existe un mont sans vallée [montem sine valle]. Il y a notamment dans la seconde partie des Principia, dans le chapitre qui traite du problème du vide absolute sumpto, une brève digression qui porte justement sur cette question:

Ut errorem illum emendemus, considerare oportet nullam quidem esse connexionem inter vas et hoc vel illud corpus particulare quod in eo continetur, sed esse maximam, ac omnino necessariam, inter vasis figuram concavam et extensionem in genere sumptam, quae in ea cavitate debet contineri. Adeo ut non magis repugnet non concipere montem sine valle, quam intelligere istam capitate absque extensione in ea contenta (AT, VIII, p. 50) ${ }^{33}$.

Si on prend la peine de confronter ce dernier extrait avec la lettre adressée à Arnaud, la différence de ton entre les deux textes ressort clairement:

Mihi autem non videtur de ulla unquam re esse dicendum, ipsam a Deo fieri non posse; cum enim omnis ratio veri et boni ab ejus omnipotentia dependeat, nequidem dicere ausim, Deum facere non posse ut mons sit sine valle, vel ut unum et duo non sint tria; sed tantum dico illus tamen mentem mihi indidissem ut a me concipi non possit mons sine valle, vel aggregatum ex uno et duo bus quod non sint tria, etc., atque talia implicare contradictionem in meo conceptu (AT, V, p. 224).

Le passage qu'on vient de rapporter confirme à quel point la réflexion de Descartes est stratifiée, tout en mettant en relief la nécessité d'intégrer ses considérations métaphysiques avec cette doctrine sur les vérités éternelles qui en a influencé l'articulation. Nous pouvons donc nous interroger de nouveau sur les motifs qui ont poussé le philosophe à ne pas discuter des présupposés de sa théorie dans les écrits qu'il a décidé de publier. A notre avis, le choix cartésien résulte proprement d'une exigence stratégique: si Descartes n'avait pas agi de manière à laisser de côté les potentialités qu'il prête à Dieu dans sa doctrine, il n'aurait jamais pu obtenir une connaissance universellement garantie, un savoir capable de s'imposer en dépit de la voluntas Dei. En effet, à l'intérieur d'un système gouverné

33 Voir également AT, VII, p. 66. 
par ce Dieu, créateur libre et tout-puissant des vérités éternelles, aucune conquête intellectuelle n'aurait jamais pu revendiquer une objectivité qui ne se révélât, en dernière analyse, contingente. Il était donc indispensable de démasquer le Dieu Trompeur et d'abandonner toute velléité de donner forme à une description vraie du monde, pour se limiter à raconter une fable qui puisse néanmoins en décrire efficacement la mécanique. Dans cette représentation baroque et dans le clair-obscur de ses nombreux plis, la nature claire et distincte de nos formulations conceptuelles finit ainsi par être accompagnée d'une ombre portée sur l'essentia, elle-même obscure, de Dieu. Comme le suggère Jean-Pierre Cavaillé, Descartes instaure par conséquent avec son public de lecteurs ce même type de rapport que 'son' Dieu entretient avec ses créatures ${ }^{34}$. A l'instar de ce Deus qui potest omnia, qui ne consigne pas aux hommes les clés de son misterium et ne gomme pas la distance qui le sépare de sa théophanie, Descartes, de même, utilise l'artifice du théâtre pour représenter, dans le clair-obscur d'une proportio non inclusive, la fable de son cheminement cognitif.

La fin semble donc se relier avec le début. Après avoir assisté à la mise en scène du drama de la raison moderne par Descartes, après avoir identifié les masques de son théâtre et entendu sa fabula, nous pouvons finalement nous reporter à ces Cogitationes Privatae qui semblent être le centre névralgique de la pensée cartésienne. Larvatus prodeo: il s'agit de se déplacer masqués sur la scène de ce monde, en changeant de temps à autre de masques et en laissant à chaque acteur le loisir d'interpréter son propre rôle jusqu'au bout. Dans le cadre de cette illusion, de cette cathédrale de lumière encerclée par une ombre qui peut à tout moment interrompre l'entier spectacle, il n'y a pas d'autre remède que celui de raconter, et de se raconter, une fable. On voit ainsi que la fable est encore, et précisément au seuil de la modernité, indispensable à l'élaboration du discours philosophique. Ce n'est donc pas par hasard si, dans un portrait réalisé par Jean-Baptiste Weenix, le philosophe est immortalisé tenant en main un livre entrouvert où l'on peut lire, malgré l'ombre portée sur la page: Mundus est fabula.

34 "Descartes fait tout pour instaurer avec son public un rapport similaire à celui que Dieu entretient avec ses créatures. Dieu se sonne à connaître sans se donner à comprendre, institue librement les vérités tout en protégeant le mystère de sa grandeur et de sa puissance, comme un monarque veille à préserver sa majesté en évitant de se rendre familier à ses sujets", J.-P. Cavaillé, Descartes. La fable du monde, cit., p. 297. 